\title{
Metabolic modeling predicts specific gut bacteria as key determinants for Candida albicans colonization levels
}

\author{
Mohammad H. Mirhakkak ${ }^{1}$. Sascha Schäuble $\mathbb{1}^{1}$ - Tilman E. Klassert ${ }^{2}$. Sascha Brunke ${ }^{3}$. Philipp Brandt ${ }^{4}$. \\ Daniel Loos $\mathbb{D}^{1}$ - Ruben V. Uribe ${ }^{5}$. Felipe Senne de Oliveira Lino ${ }^{5}$ - Yueqiong $\mathrm{Ni}^{1}$. Slavena Vylkova ${ }^{4}$. \\ Hortense Slevogt ${ }^{2} \cdot$ Bernhard Hube $^{3,6} \cdot$ Glen J. Weiss $^{7} \cdot$ Morten O. A. Sommer $\mathbb{D}^{5} \cdot$ Gianni Panagiotou (iD) ${ }^{1}$
}

Received: 15 April 2020 / Revised: 6 November 2020 / Accepted: 18 November 2020 / Published online: 15 December 2020

(c) The Author(s) 2020. This article is published with open access

\begin{abstract}
Candida albicans is a leading cause of life-threatening hospital-acquired infections and can lead to Candidemia with sepsislike symptoms and high mortality rates. We reconstructed a genome-scale $C$. albicans metabolic model to investigate bacterial-fungal metabolic interactions in the gut as determinants of fungal abundance. We optimized the predictive capacity of our model using wild type and mutant $C$. albicans growth data and used it for in silico metabolic interaction predictions. Our analysis of more than 900 paired fungal-bacterial metabolic models predicted key gut bacterial species modulating $C$. albicans colonization levels. Among the studied microbes, Alistipes putredinis was predicted to negatively affect $C$. albicans levels. We confirmed these findings by metagenomic sequencing of stool samples from 24 human subjects and by fungal growth experiments in bacterial spent media. Furthermore, our pairwise simulations guided us to specific metabolites with promoting or inhibitory effect to the fungus when exposed in defined media under carbon and nitrogen limitation. Our study demonstrates that in silico metabolic prediction can lead to the identification of gut microbiome features that can significantly affect potentially harmful levels of $C$. albicans.
\end{abstract}

\section{Highlights}

- Genome-scale model reconstruction of C. albicans with $89 \%$ growth accuracy.

- Mutualism and parasitism are the most common predicted C. albicans-gut bacteria interactions.

- Metagenomic sequencing and in vitro assays reveal modulators of fungal growth.

- Alistipes putredinis potentially prevents elevated C. albicans levels.

These authors contributed equally: Mohammad H. Mirhakkak, Sascha Schäuble

Supplementary information The online version of this article (https:// doi.org/10.1038/s41396-020-00848-z) contains supplementary material, which is available to authorized users.

$\triangle$ Gianni Panagiotou

Gianni.Panagiotou@leibniz-hki.de

$1 \quad$ Systems Biology \& Bioinformatics Unit, Leibniz Institute for Natural Product Research and Infection Biology - Hans Knöll Institute, 07745 Jena, Germany

2 ZIK Septomics, Jena University Hospital, 07745 Jena, Germany

3 Department of Microbial Pathogenicity Mechanisms, Leibniz Institute for Natural Product Research and Infection Biology Hans Knöll Institute, 07745 Jena, Germany

\section{Introduction}

The fungus Candida albicans is found on the mucosal surfaces of at least $50-70 \%$ of healthy adults [1] and is a classic opportunistic pathogen. It resides as a harmless commensal but can become pathogenic in immunocompromised patients or under microbial dysbiosis $[2,3] . C$.

4 Septomics Research Center, Friedrich Schiller University, Leibniz Institute for Natural Product Research and Infection Biology Hans Knöll Institute, 07745 Jena, Germany

5 Novo Nordisk Foundation Center for Biosustainability, Technical University of Denmark, 2800 Kgs. Lyngby, Denmark

6 Institute for Microbiology, Friedrich Schiller University, 07743 Jena, Germany

7 MiRanostics Consulting, 85755 Oro Valley, AZ, USA 
albicans causes up to 300,000 deaths per annum worldwide with an increasing number of individuals at risk [4]. Therefore, efforts to understand drivers of commensal or pathogenic outcomes of this fungus have intensified.

Recent studies found links between alterations in the composition and functionality of the gut microbiota and development of local or systemic $C$. albicans infections $[5,6]$. A microbial tryptophan metabolic pathway appears to preserve immune physiology at mucosal surfaces by promoting indole-3-aldehyde production that contributes to IL-22 transcription [7]. Other gut microbial products such as bacteriocin are directly active against C. albicans [8]. A study of rectal samples from a cohort of 150 children linked gut microbiota to Candida prevalence, with a relative reduction in Candida species in children who received probiotics along with broad spectrum antibiotics [9]. Neither the dynamics of Candida species in the human gut nor the specific microbial contributors to the observed reduction have been studied, however. Based on these diverse findings, the commensal status of $C$. albicans appears to be related to the global taxonomy and functionality of the host microbiome.

A promising approach to analyzing interactions between C. albicans and gut microbial species uses genome-scale metabolic models (GSMMs) GSMMs have improved the biotechnological productivity of bacteria [10-12], revealed plant metabolic processes [13], and elucidated the Crabtree effect in yeast [14-16] and the Warburg effect in cancer cells [17]. Recent pioneering studies have developed highquality GSMMs for gut bacteria that enable in silico analysis of gut metabolic functions and interactions [18, 19]. These resources have advanced the study of gut microbes and their respective pairwise interactions but have not yet been used to study interactions with opportunistic fungal pathogens such as $C$. albicans. The potential of gut microbes to influence the overall fitness of the fungus must be elucidated to support development of prophylactic or therapeutic strategies to control $C$. albicans.

We constructed a GSMM of $C$. albicans, starting with an automatically generated template model [20, 21]. We substantially improved its performance with manual curation and adaptation to phenotype microarray experiments. We used both, publicly available data [22] and new phenotypic microarray data for both wild type and mutant $C$. albicans strains. Our model predictions surpassed those of other GSMMs for species closely related to $C$. albicans that could serve as proxies for this fungus. We used the GSSM to simulate in silico pairwise metabolic interactions between C. albicans and each of 910 gut bacteria models. We challenged our predictions in vitro by growing $C$. albicans in carbon or nitrogen limited defined media in the presence of predicted fungal growth affecting metabolites. We further validated our results with stool samples from 24 human subjects, using metagenomics and internal transcribed spacer (ITS) sequencing to identify bacterial species associated with significant effects on $C$. albicans metabolism and growth. Finally, we assessed fungal growth in bacterial spent media experiments.

\section{Materials and methods}

\section{Model reconstruction}

To generate the $C$. albicans GSMM, we used the $C$. albicans metabolic model automatically reconstructed by the CoReCo pipeline as a template. In brief, CoReCo combines information from multiple data sources into a unified database and evaluates the probability of any reaction occurring in the target organism by computing a score for each enzyme based on sequence homology [20]. We refined the model in four consecutive steps (Fig. 1A) that included the identification and removal of duplicate metabolites, determination and resolve of erroneous energy-generating cycles [23], adaptation to phenotypic microarray data and exchange reaction modification based on flux variability analysis (FVA, Supplementary Material, Supplementary Data S1) [24].

\section{Pairwise simulations}

Pairwise simulations adapted from Heinken and Thiele [25] were performed using 818 AGORA 1.03 GSMMs [18] downloaded from (https://www.vmh.life) and 92 CarveMe gut bacterial GSMMs [19]. In brief, the C. albicans GSMM was paired to individual bacterial GSMMs and subsequently optimized by simultaneously maximizing C. albicans and bacteria biomass reactions. Interaction type was determined by taking the optimized growth rate in the pair compared to the growth rate of the individual GSMM into account (Supplementary Material).

To evaluate dissimilarities between promoters and inhibitors of $C$. albicans from different phyla, Bray-Curtis distances were calculated based on flux distributions of individual bacteria from pFBA simulations on different media [18] (https://www.vmh.life, Supplementary Table S1). Fisher's exact tests were calculated to determine if a specific phylum was enriched with species that either inhibited or promoted $C$. albicans growth. The python code and metabolic models for simulating pairwise GSMMs are available at https://github.com/mohammadmirhakkak/Ca ndida-albicans-microbiome-interaction.

\section{Phenotypic microarray experiments}

C. albicans wild type and multiple mutant strains (Table 1) were pre-grown on YPD (1\% yeast extract, $2 \%$ peptone, 


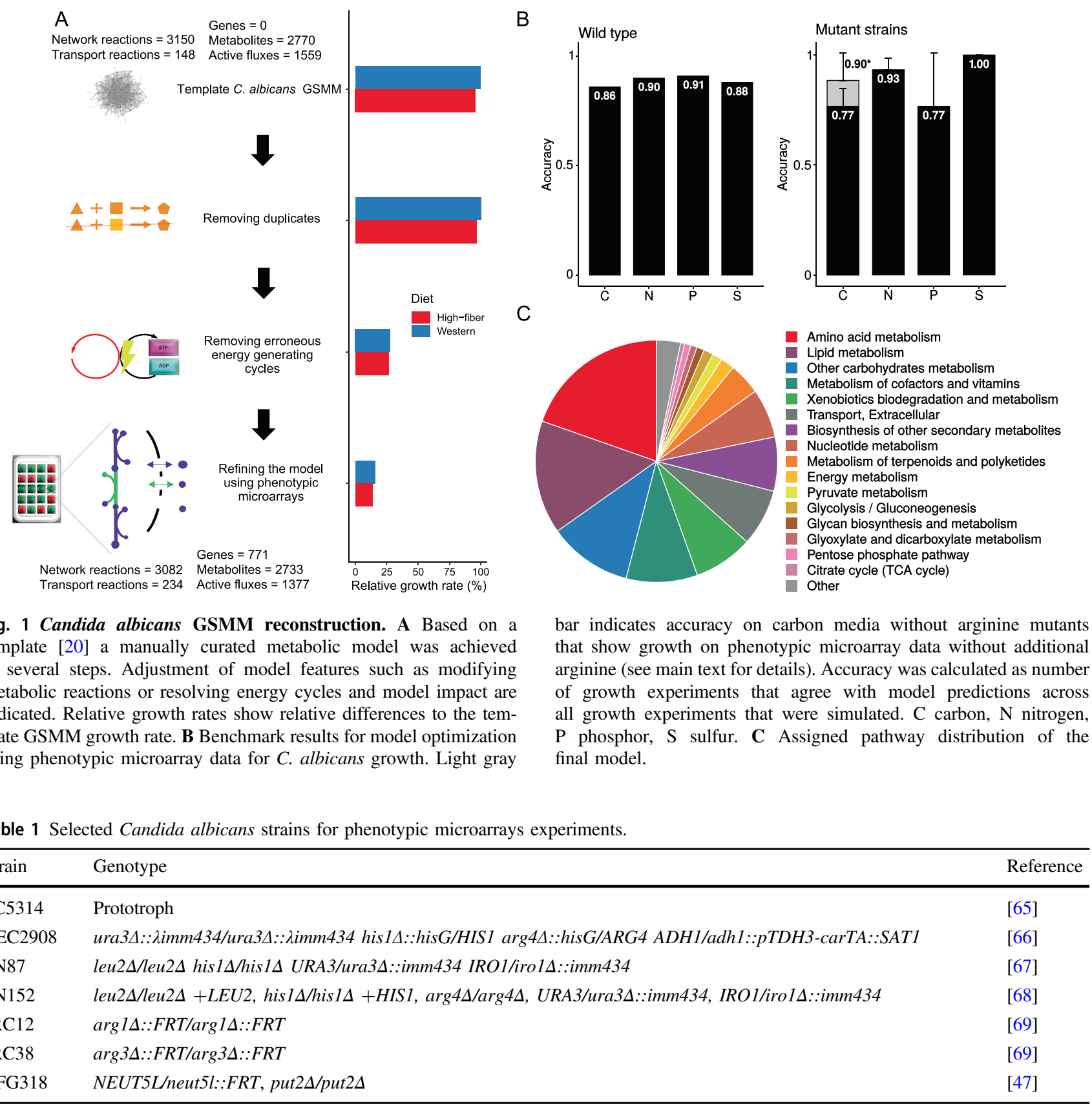

$2 \%$ glucose, $2 \%$ agar) plates. Phenotype microarrays were performed by using microarray plates, reagents and devices according to the manufacturer Biolog, Inc. (Hayward, CA, USA) instructions. Briefly, C. albicans cells were taken directly from YPD agar plates and diluted in sterile $\mathrm{dH} 2 \mathrm{O}$ to $62 \%$ transmittance as measured by a turbidimeter (Biolog, Inc.). Next, cells were combined with inoculating fluid IFY-0 base (1.2x), redox dye mix D (75x) (Biolog Inc.), and further supplemented with either Glucose, Lglutamic acid, potassium phosphate or sodium sulfate (Sigma-Aldrich) were required. $100 \mu \mathrm{l}$ of the respective mixture $(83.33 \%$ IFY-0 base, $1.33 \%$ redox dye D, $8.33 \%$ supplements, $2.08 \%$ cells, and if required $3.12 \%$ Glucose and $\mathrm{dH} 2 \mathrm{O}$ ) was added to each well of a Biolog Phenotype Microarray 96-well plate for fungi to test for metabolic activity in the presence of carbon sources (PM1 and PM2A), nitrogen sources (PM3B), and phosphorus and sulfur sources (PM4A). The phenotype microarrays were incubated at $30^{\circ} \mathrm{C}$ in an OmniLog multiple plate reader in order to prevent hyphae formation that otherwise perturb growth measurements. Reduction of the redox dye, an indicator for metabolic activity, was measured kinetically every $15 \mathrm{~min}$ at an OD of $750 \mathrm{~nm}$ for $24-48 \mathrm{~h}$. Each experiment was performed twice. Data analysis was done 
using the $\mathrm{R}$ opm package for OmniLog phenotype microarray datasets [26].

\section{In vitro experiments}

\section{Growth of C. albicans in presence of metabolites}

To determine the effect of the metabolites on C. albicans growth, the clinical isolate SC5314 was grown overnight at $30{ }^{\circ} \mathrm{C}$ in YPD complex medium $(1 \%$ yeast extract, $2 \%$ peptone, $2 \%$ glucose). $30{ }^{\circ} \mathrm{C}$ were chosen to prevent hyphae formation provoked at higher temperatures, which otherwise perturb growth curve measurements. Yeast cells were washed three times with sterile $\mathrm{H}_{2} \mathrm{O}$ by centrifugation for $5 \mathrm{~min}$ at $4,200 \times \mathrm{g}$. Test medium was composed of $1 \times$ yeast nitrogen base (YNB, Formedium) with either (standard) $0.25 \% \mathrm{NH}_{4} \mathrm{SO}_{4} / 2 \%$ glucose, (C limited) $0.25 \%$ $\mathrm{NH}_{4} \mathrm{SO}_{4} / 0.25 \%$ glucose, (N limited) $0.008 \% \mathrm{NH}_{4} \mathrm{SO}_{4} / 2 \%$ glucose, or (C/N low) $0.016 \% \quad \mathrm{NH}_{4} \mathrm{SO}_{4} / 0.5 \%$ glucose. Test substances were obtained from Sigma-Aldrich and were dissolved in $\mathrm{H}_{2} \mathrm{O}$ at (nitrite) $156 \mathrm{mM}$, (desoxyadenosine) $100 \mathrm{mM}$, (sodium formate) $1 \mathrm{M}$, (putrescine) $500 \mathrm{mM}$, (L-asparagine) $100 \mathrm{mM}$, or (L-proline) 15.6 $\mathrm{mM}$. Assays were performed as 1:2 dilution series in 96 well plates (TPP, flat bottom) and were composed of $180 \mu \mathrm{l}$ test medium, $10 \mu \mathrm{l}$ test substance, $10 \mu \mathrm{l}$ yeast solution (1:10 dilution in $\mathrm{H}_{2} \mathrm{O}$, final $\mathrm{OD}_{600}$ of 0.1 ). Initial $\mathrm{pH}$ was verified to be at the expected $\approx 5.8$. Growth was followed over at least $24 \mathrm{~h}$ using a Tecan infinite 200 multiwell plate reader set to $30^{\circ} \mathrm{C}$, with measurements at $600 \mathrm{~nm}$ every $15 \mathrm{~min}$ following $10 \mathrm{~s}$ of orbital shaking. All measurements were performed in triplicate from independent overnight cultures at different days. Growth was evaluated as area under the curve (AUC, trapezoidal method using GraphPad prism 8.1.2; baseline at mean of first three measurement) over $24 \mathrm{~h}$ and expressed as percent change compared to control setups $\left(\mathrm{H}_{2} \mathrm{O}\right.$ instead of test substance) in the same medium. AUCs were determined for three replicates, and mean change compared to controls is shown with standard deviations (SD) as error bars.

\section{Strains and culture conditions}

A. putredinis (DSM17216), B. ovatus (ATCC 8483), B. vulgatus (ATCC8482), E. lenta (DSM2243), P. copri (DSM18205), R. torques (ATCC27756), E. coli (MG1655 and BAA-1161), P. corporis (DSM18810), C. albicans (SC5314/ATCC MYA-2876), C. albicans (ATCC 10231) and C. albicans (ATCC 18804) were grown at $37^{\circ} \mathrm{C}$ under anaerobic conditions (gas mixture, $95 \% \mathrm{~N}_{2}$ and $5 \% \mathrm{H}_{2}$ ) in prereduced modified GAM (mGAM, Nissui Pharmaceutical Co. Ltd.) broth for liquid cultures or broth supplemented with agar for growth on plates. $37{ }^{\circ} \mathrm{C}$ and anaerobic conditions were chosen to resemble best the natural environment of these gut bacteria.

\section{Sterile bacterial spent media}

Bacterial strains were grown for $48 \mathrm{~h}$ in GAM broth, then subcultured 1:50 in fresh GAM broth and grown for $48 \mathrm{~h}$ in anaerobic conditions at $37{ }^{\circ} \mathrm{C}$ to resemble the gut environment. Cultures were centrifuged at $11,000 \times g$ for $5 \mathrm{~min}$ and spent media removed without disturbing the pellet. Spent media were passed through a $0.2 \mu \mathrm{m}$ syringe filter to remove remaining bacteria. After filtration, the $\mathrm{pH}$ of the spent media was analyzed using an electronic $\mathrm{pH}$-meter (Supplementary Table S2). 1\% (v/v) of Phosphate-buffered saline (PBS) was added in each experiment to maintain a constant $\mathrm{pH}$.

\section{Growth assays}

An overnight culture of $C$. albicans was grown aerobically in mGAM media at $37^{\circ} \mathrm{C}$. Aerobic conditions were chosen to enable sufficient growth of $C$. albicans. Cells were then subcultured at a 1:1000 dilution into $150 \mu \mathrm{l}$ of sterile spent bacterial media in different proportions: 75 and 100\%. The spent media were diluted in fresh $25 \%$ mGAM broth for $75 \%$ spent media proportion and PBS (1\% v/v), accordingly. The fermentation was performed in flat-bottom, 96well plates. The plates were incubated for $24 \mathrm{~h}$ at $37^{\circ} \mathrm{C}$, with continuous orbital shaking at $900 \mathrm{rpm} .37^{\circ} \mathrm{C}$ did not induce notable hyphae formation in growth assays and therefore did not perturb growth measurements on spent media. Cell densities were measured every $10 \mathrm{~min}$ at optical density $600 \mathrm{~nm}$ (OD600) using a microtiter reader (BioTek ELx800). Growth rates were calculated by plotting log of OD measurements in log phase and calculating the slope of the time points in log phase where $r^{2}$ was closest to 1 , using at least 12 time points over $2 \mathrm{~h}$. Growth inhibition was determined as the growth rate of $C$. albicans in spent bacterial media normalized to the growth rate in the corresponding mGAM fresh media dilution.

\section{Microbiome profiling}

Bacterial and fungal species profiles were generated for stool samples from a human cohort of 24 individuals at Western Regional Medical Center, Goodyear, Arizona, USA. Samples were collected after signed informed consent under a protocol approved by the Western Institutional Review Board (WIRB protocol number 20140271, Pallyup, Washington, USA). All subjects had been diagnosed with different types of cancer, with heterogeneous stage, treatment, and histological findings. Metagenomic sequencing 
was performed at BGI, Hong Kong S.A.R., China, as described in Qin et al. [27], and ITS amplicon sequencing of the mycobiome was performed at ZIK Septomics, University Hospital Jena, Thuringia, Germany (extended details in Supplementary Material). Bacterial reads were obtained using 150-bp Illumina PE whole metagenome sequencing. Species profiling was by MetaPhlAn 2.7.6 [28]. after applying an in-house pipeline for quality control [29] and removal of human reads. Fungal reads were obtained using 250-bp Illumina PE ITS1 amplicon sequencing (extended details in Supplementary Material). The DADA2 ITS Pipeline Workflow 1.8 was followed for amplicon sequence variants [30]. Mothur classifier [31] called from QIIME 1.9.1 [32] and the UNITE database 7.2 [33] were used for fungal taxonomic assignments. Bacterial species were considered for further analysis, if a GSMM model was available, the species was part of the Open Tree of Life 10.4 [34] and it was prevalent in at least 4 of 26 samples. Correlations of bacterial abundance and growth rates to $C$. albicans abundance were tested using two-sided Spearman correlation $(p<0.05)$. The bacterial and fungal profiles are available under the ENA Study Nr. PRJEB33756.

Partial spearman correlation was computed within $\mathrm{R}$ using the package PResiduals (v0.2-6).

\section{Ordinal regression model}

Relative abundance values for $C$. albicans from human samples were grouped into two sets determined by a $20 \%$ relative abundance threshold. To predict $C$. albicans abundance levels, three binomial ordinal regression models were generated using as independent variables the bacterial abundance values, predicted interaction coefficients, and products of multiplying abundance values by predicted interaction coefficients. For model building, we either selected five bacterial species that significantly correlated with $C$. albicans by relative abundance and two that significantly correlated by GRiD value, or selected bacteria based on whether they increase performance as described in the main text. The predictive power of each model was assessed by determining the true/false positive/ negative and accuracy values and by analyzing receiving operating characteristic curves using the R package ROCR (ver. 1.0-7).

\section{Results and discussion}

\section{Reconstruction of a Candida albicans genome-scale metabolic model}

To develop a $C$. albicans GSMM, we started with a model automatically generated by the Comparative
ReConstruction (CoReCo) pipeline [20]. The initial $C$. albicans CoReCo model (BioModels ID 1604280052) comprised 2770 metabolites and 3298 reactions, of which 3150 were network and 148 transport reactions (Fig. 1A). In addition to a unique reaction set, the initial CoReCo GSMM for $C$. albicans contained multiple nontrivial duplicate reactions and metabolites. Typically, these involved marginally differing metabolite names such as L-Glutamate and Glutamate that had not been automatically detected by the CoReCo platform (Fig. 1A, Supplementary Data S1, Tables S1, S3, and S4).

We also curated energy-generating cycles (EGCs) that created energy compounds such as ATP without requiring nutrient uptake [23]. We resolved these infeasible EGCs by identifying and correcting implausible reaction directionalities using metabolic pathway databases such as BioCyc (Supplementary Table S4). For example, in our initial $C$. albicans model, we found an ATP-producing EGC that involved phosphate rather than pyrophosphate as indicated by BRENDA, Biocyc and KEGG databases. Correcting the involved reaction acetoacetate:CoA ligase resolved this particular EGC and additional EGCs while maintaining a viable biomass flux. We corrected six reactions by either changing metabolite usage or reaction directionality based on KEGG or BioCyc information (Supplementary Table S4). Resolving EGCs also reduced the flux through the biomass reaction towards 1.4 , which is closer to the biomass reaction flux for other fungal models such as the yeast consensus model [35, 36] (Fig. 1A).

Next, we adapted our model to multiple phenotypic microarray growth experiments of $C$. albicans. These comprise up to 1440 different defined media experiments with diverse carbon, nitrogen, phosphorus or sulfate sources. We used a publicly available dataset with different $C$. albicans phenotypes [22] and created additional phenotypic microarray growth experiments including several mutant strains for e.g., different arginine biosynthesis steps (see Methods for details). Of the metabolites in the dataset, 455 mapped to metabolites in our $C$. albicans GSMM. First, we adapted our model to growth experiments that were in agreement between data from Ene et al. [22] and our own prototrophic C. albicans wild type strain SC5314 dataset for C. albicans. Of note, using only compatible growth measurements ensured robust growth information across different temperatures applied in our and in the published data by Ene et al. [22] (cf. Materials and Methods). Second, we adapted our model to all phenotypic growth experimental data of $C$. albicans mutant strains for those growth conditions that yielded the same results for both our wild type and the Ene et al. dataset in the prior step (Supplementary Table S4). These refinement steps included, for example, enabling ammonia production from urea or via lysine degradation, which was not initially present in our model 
(Supplementary Tables S4 and S5). Based on the growth data, we added 107 potentially feasible exchange reactions involving metabolites associated with $C$. albicans growth. By applying flux variability analysis [24] we also identified five exchange reactions that shuttle 4-aminobenzoate, folinic acid, 7,8-diaminononanoate, hexadecanal, and hydrogensulfite in and out of the network, but do not support growth and removed these from the GSMM accordingly. Overall, we achieved a high compatibility between our model predictions and the phenotypic microarray growth experiments with nitrogen and sulfur predictions reaching above 90\% accuracy over all mutant experiments (Fig. 1B, Supplementary Table S4). Of note, model predictions for carbon source experiments of three different $C$. albicans mutants for arginine biosynthesis $(\arg 1 \Delta, \arg 3 \Delta$ and $\arg 4 \Delta$ ) predicted no growth rate for all carbon sources, since arginine is essential for the biomass objective function. Surprisingly, up to $33 \%$ of the associated phenotypic microarray data showed fungal growth, despite the inability to synthesize arginine due to the knock out. This might be due to recycling of available proteins in e.g., fungal vacuoles, as the defined growth media itself does not contain arginine, unless specifically tested. Considering only growth experiments that show no growth for arginine biosynthesis mutant strains our model accuracy for carbon sources reaches $90 \%$ across all tested $C$. albicans mutant (Fig. 1B).

Finally, we added gene annotation for $\sim 1500$ reactions and associated individual gene to reaction rules using the KEGG database. We also added pathway association for all reactions if available and unified pathway associations across resources. This step resulted in 83 pathway definitions, including all essential pathways such as in central carbon, amino acid, and lipid metabolism (Fig. 1C). Overall, our model refinements resulted in an addition of 771 genes, led to a final model comprising 3082 metabolic reactions ( -68 compared to draft model) and 2733 metabolites ( -37 metabolites compared to draft model) and a reduced active flux flexibility towards biomass by $11.7 \%$ (Fig. 1A, Supplementary Data S1, Tables S1, S3, and S4).

\section{Pairwise growth simulations predict gut bacteria modulating essential C. albicans metabolic activity}

Next, we generated in silico metabolic interaction predictions about $C$. albicans coupled to gut bacteria GSMM models. Using 910 publicly available GSMMs for gut microbial species [18, 19], we performed pairwise metabolic analysis by linking our $C$. albicans model with each gut microbe GSMM [25] (Supplementary Table S1). The majority of GSMMs from different sources [18, 19] gave compatible growth rate predictions. To streamline further analysis, we continued with the assembly of gut organisms through reconstruction and analysis (AGORA) GSMMs (https://www.vmh.life) [18] unless models were available from both sources. In the latter case, we continued with the CarveMe model versions, since these are refined to bacterial growth data across 19 different media including extended pathway gap correction [37] and are based on a manually curated template model [19].

We simulated growth on two different media compositions that resemble typical Western and high-fiber diets (https://www.vmh.life) [18]. We identified the interaction type of each $C$. albicans-gut microbe pair by analyzing differences in predicted growth rates compared to growth rates derived from individual simulations using a flux balance analysis approach [38] (Methods, Pairwise simulations; Supplementary Material, Table S1). Predictions based on Western and high-fiber diets gave similar interactiontype distributions: mutualism (positive growth effect for both, $C$. albicans and paired bacteria) and parasitism (here, negative effect on $C$. albicans growth, positive growth effect on bacteria) were the most abundant ( $>81 \%$ of observed interaction types, Fig. 2A). Other interaction predictions included commensalism, in which both $C$. albicans and bacterial growth was promoted without negative effects on the respective paired microorganism (12\% and $17 \%$ for Western and high-fiber diets, respectively). Only a few examples of parasitism in which $C$. albicans exerted negative effects on gut bacteria or amensalism (no growth effect on $C$. albicans, negative effect on bacteria) or neutralism (no growth effect on both, C. albicans and gut bacteria) were observed. We further examined prediction accuracy with additional simulations on standard Gifu anaerobic medium (GAM), which was used for in vitro validation experiments (Supplementary Table S6). Growth simulations on GAM were feasible for only 200 GSMM models, as all other models yielded no biomass formation. GAM simulations identified parasitism with negative effects on bacteria for up to $16 \%$ of viable bacteria models, while parasitism with negative effects on C. albicans (53\%) and mutualism (28\%) were again the most commonly occurring interaction types (Fig. 2A). Our interaction-type analysis based on predicting growth rate differences for microorganisms in individual and paired model setups hints that the majority of gut bacteria have either a mutualistic or a parasitic relationship with $C$. albicans irrespective of medium.

To analyze if predicted interactions that promoted or inhibited $C$. albicans growth were phylum- or diet-specific effects, we performed non-metric multidimensional scaling analysis (NMDS) based on parsimonious flux balance analysis (pFBA)-derived values for the top 50 promoting and inhibiting bacterial species [39] (Supplementary Table S1). The predicted top 50 inhibiting bacterial species comprised mainly species from the genus Bifidobacterium 
A

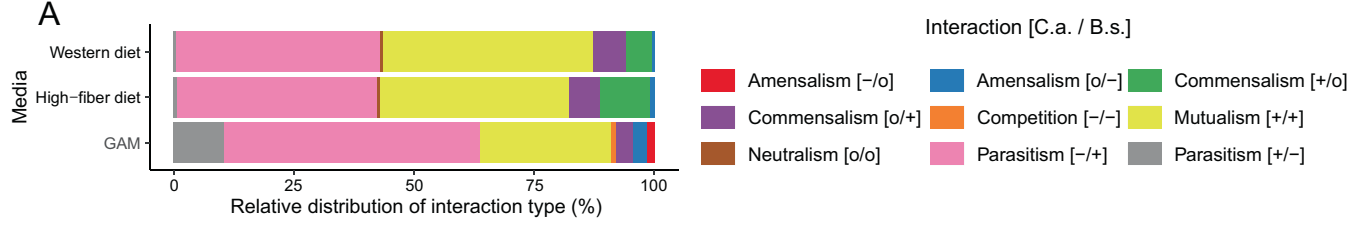

B

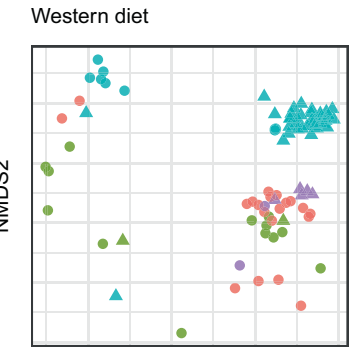

NMDS1

Interaction - Inhibitor \& Promoter

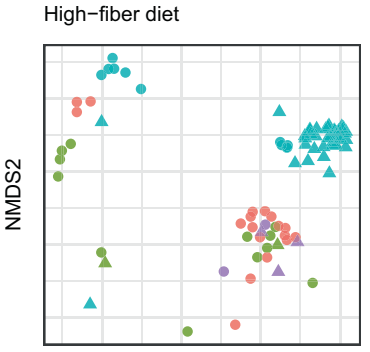

NMDS1

Phylum Actinobacteria Bacteroidetes

C
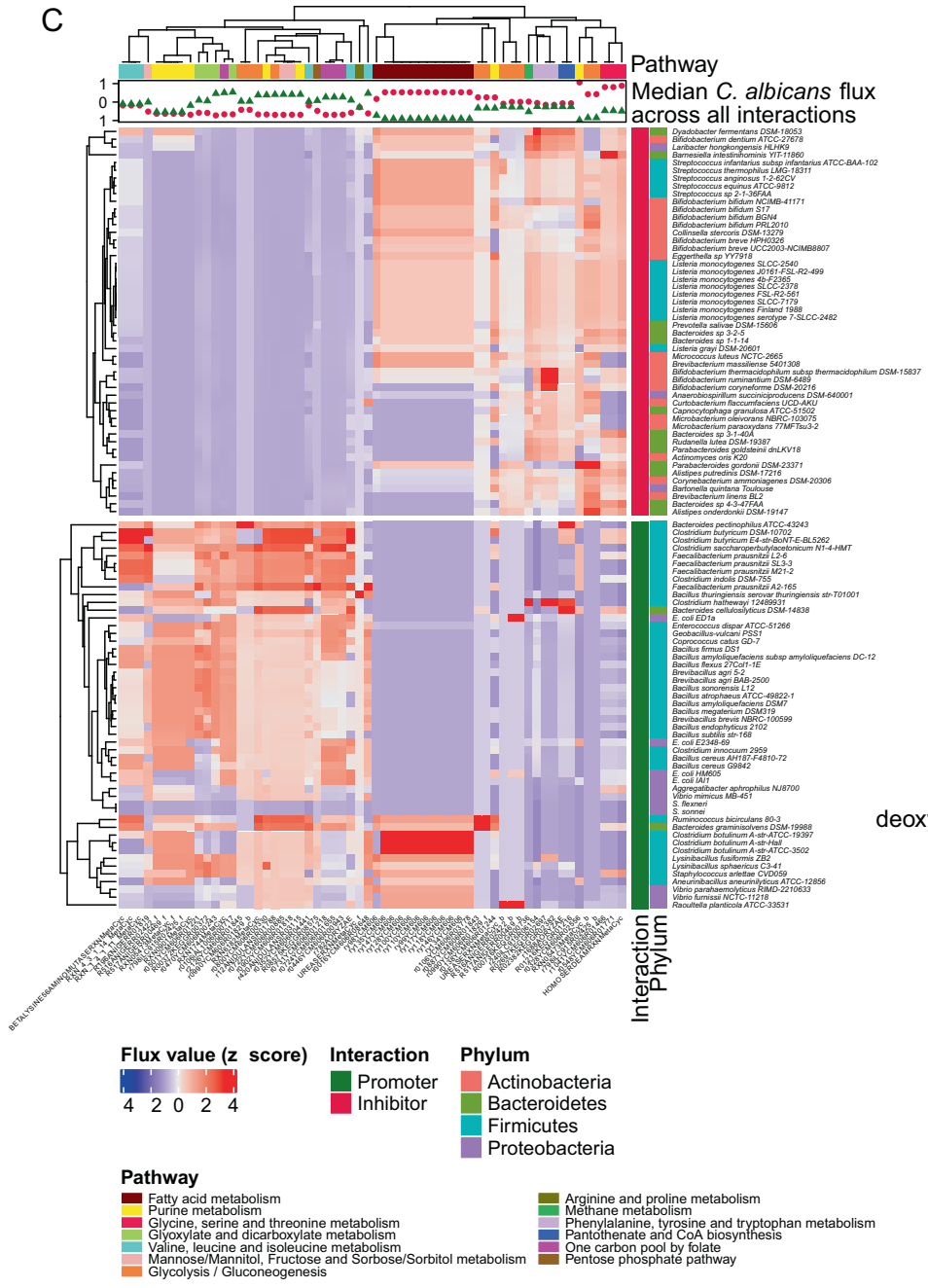

D

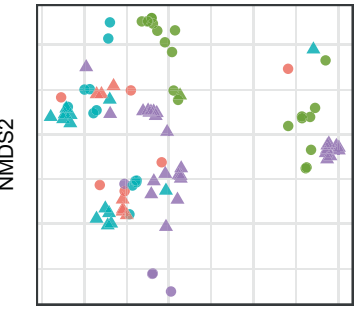

NMDS1

Firmicutes Proteobacteria

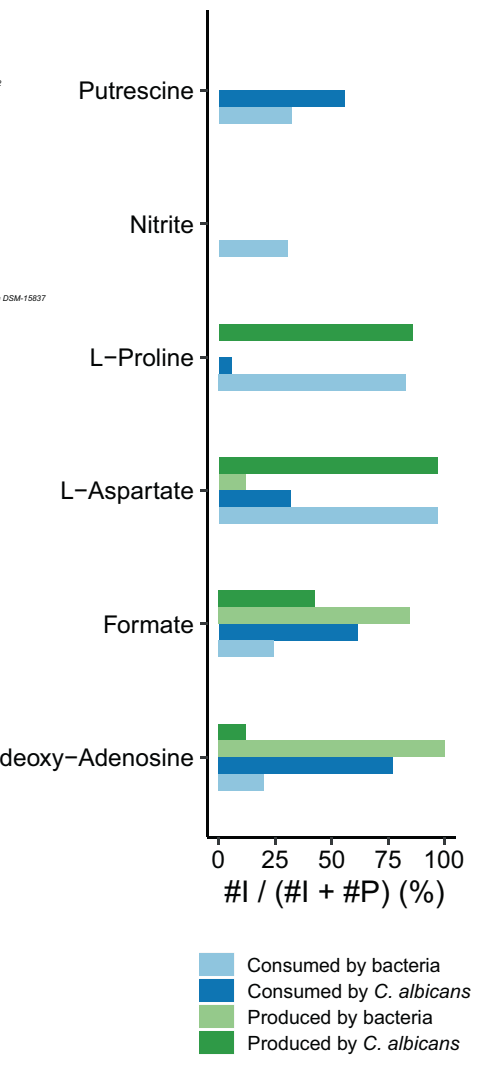

and Listeria, but also included several bacteria from the Clostridium or Vibrio genus (Supplementary Table S1). After removal of a few outliers (up to seven GSMMs comprising several Escherichia coli strains, among others) we observed distinct grouping of the Firmicutes phylum on contrast, the predicted top $50 \mathrm{C}$. albicans promoting bacteria included many bacteria with the Bacillus, Bacteroides, 
Fig. 2 Pairwise in silico interaction experiments. A Distribution of interaction type for C. albicans (C.a.) and bacterial species (B.s.). Interactions have positive (+), negative (-) or no (o) effect on growth rates of fungus or bacteria as indicated for interaction types. B Nonmetric multidimensional scaling (NMDS) plots of bacterial reaction flux rates for top $50 \mathrm{C}$. albicans-inhibiting and -promoting bacteria simulated for three different media (Western and high-fiber diet, Gifu anaerobic media (GAM)). C Metabolic reactions of $C$. albicans with the most substantially differing flux rates of $C$. albicans when paired with top 50 inhibiting or promoting bacteria. Top: median $C$. albicans flux rate differences across all bacterial species paired with $C$. albicans. D Analysis for selected metabolites based on exchange reaction fluxes of simulated fungal-bacterial pairs for top 50 promoting or inhibiting bacterial species (cf. Supplementary Table S5). $x$-axis indicates the percentage of exchange reaction fluxes with bacteria that inhibit C. albicans growth.

both Western and high-fiber diets in the NDMS plots, with a largely predicted positive effect on $C$. albicans growth (Fig. 2B). The majority of Proteobacteria (for $71 \%$ of the respective GSMMs on Western, and $73 \%$ on high-fiber diet) were predicted to show a positive effect on $C$. albicans growth as well and show similar flux distributions to some Bacteroidetes and most Actinobacteria. In contrast, the predicted impact of Bacteroidetes was primarily negative (for $87 \%$ GSSMs on Western, and $86 \%$ on high-fiber diet) on $C$. albicans growth with differing flux distributions for both, Western and high-fiber diet (Fig. 2B). All Actinobacteria were predicted to inhibit $C$. albicans growth as well and showed similar flux distributions to some Bacteroidetes and most Proteobacteria for Western and high-fiber diets. Of note, in silico simulations on GAM revealed notable differences of flux distributions compared to Western or high-fiber diet (e.g., 53\% of the paired Actinobacteria showed promoting effects on $C$. albicans) showing that GAM based simulations differ to some degree from these diets (Fig. 2B). The predicted interaction type was significantly dependent on phyla (chi-squared test, $p$ value $=$ $2.5 \mathrm{e}-9$ on Western diet) with Actinobacteria (false discovery rate-corrected Fisher's exact test $p$ value $=7.0 \mathrm{e}-7$ ) and Firmicutes (false discovery rate-corrected Fisher's exact test $p$ value $=1.5 \mathrm{e}-5$ ) primarily responsible for the interaction type. Altogether, our analyses suggested that under Western and high-fiber diets, species from specific phyla had positive (Firmicutes, Proteobacteria), or negative (Actinobacteria, Bacteriodetes) effects on $C$. albicans growth with distinct flux distributions particularly for predicted growth-promoting Firmicutes species.

Next, we investigated $C$. albicans-specific reaction fluxes derived from paired fungal-bacterial model simulations. Paired bacteria were again selected based on their predicted impact on C. albicans growth rates such that its difference between paired fungal-bacterial and individual model simulations is most pronounced and comprised bacteria from four different phyla (Fig. 2C, Supplementary Fig. S1). From the obtained flux distributions $C$. albicans reactions were selected based on the most pronounced flux differences between $C$. albicans paired with predicted growth inhibiting and growth-promoting bacterial GSMMs (Methods, Pairwise simulations). Of note, predicted median flux differed notably for many reactions comparing the top inhibiting and promoting bacteria to all paired gut microbes (Fig. 2C, upper panel). These changes were particularly present in sugar, fatty acid, folate, and small amino acid associated pathways, but also in glycolysis and hint towards a shifted flux in the selected bacteria that dominantly affect C. albicans growth. Altered reaction fluxes were identified across major pathways including carbon, amino acid, purine and fatty acid metabolism. Central metabolites such as alpha-ketoglutarate, pyruvate and glutamate were balanced towards net production or consumption, depending on the paired gut microbe in the in silico simulation. C. albicans growth is affected by modulating carbohydrate metabolism [40] or the availability of amino acids such as leucine or valine [41] and might be used by gut microbiota to prevent or promote $C$. albicans growth. Specifically, we predicted elevated reaction fluxes that consumed L-glutamate in $C$. albicans when paired with fungal growth-promoting bacteria via the aminotransferases Glycine:2-oxoglutarate aminotransferase and branched-chain amino acid aminotransferase. Aminotransferases were studied before in the context of Candida infection and were attributed to the nutritional versatility of Candida species [42, 43]. Moreover, glutathione synthesized from L-glutamate is important in counteracting oxidative stress [44]. Predicted reaction fluxes including amino acids were notably different among top promotors and inhibitors and may serve as potential targets for identifying antifungal agents [45]. These findings suggest that gut bacteria that potentially perturb $C$. albicans growth may cause metabolic shifts in $C$. albicans towards L-glutamate-promoting reactions, which may allow the fungus to evade harmful oxidative stress levels.

Next, we focused on investigating fluxes of metabolite exchange reactions. Exchange reactions allow to shuttle metabolites in and out of a joint compartment in our paired in silico models. These joint compartments serve as a connection between the respective bacterial and the fungus model and allow to predict potentially $C$. albicans growth rate influencing metabolites (Fig. 2D, Supplementary Table S7). To identify such metabolites we specifically filtered for exchange reaction fluxes of metabolites that are primarily present for either $C$. albicans growth inhibiting or promoting bacteria as derived from our paired fungal-bacterial model simulations. Again, many amino acids like L-proline, or L-aspartate, but also other factors such as nitrite or putrescine are predicted to be notably differentially consumed by fungus or bacteria. 


\section{In vitro experiments and metagenomics analyses support metabolic dependencies of $C$. albicans}

To test the quality of our in silico analysis, we investigated C. albicans growth in the presence of metabolites, performed metagenomic sequencing for 24 individuals and assessed $C$. albicans growth in bacterial spent media.

First, we grew $C$. albicans in the presence of metabolites and investigated the growth-promoting or -inhibiting effect of these metabolites under different carbon and/or nitrogen availabilities (Fig. 3A). We selected six metabolites that were either primarily consumed or produced by either fungus or bacteria in our paired metabolic in silico simulations (Fig. 2D, Supplementary Table S7). We hypothesized that metabolites that e.g., are predicted to be consumed by fungal growth inhibiting bacteria are either withheld from the fungus or cannot be metabolized by the fungus and are thus beneficial for the bacteria to outgrow $C$. albicans. Likewise, metabolites that are predicted to be produced by bacteria or consumed by the fungus when $C$. albicans is paired with growth inhibiting bacteria might provide clues of metabolites with a potential negative effect on $C$. albicans growth. Nitrite showed severe growth inhibiting effects on $C$. albicans, irrespective of available carbon and nitrogen source concentrations. The same inhibiting influence was observed to a lesser extent for putrescine and for the formic acid salt, sodium formate. Both putrescine and formic acid were also predicted to be primarily consumed by $C$. albicans when paired with fungal growth inhibiting bacteria. In contrast, L-Aspartate and
A

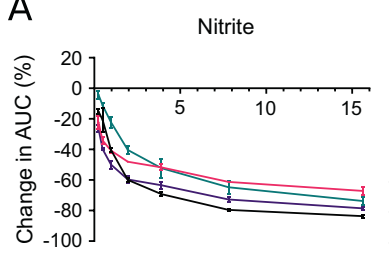

L-Aspartate

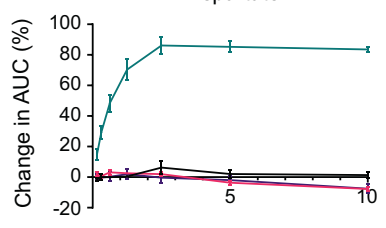

Putrescine

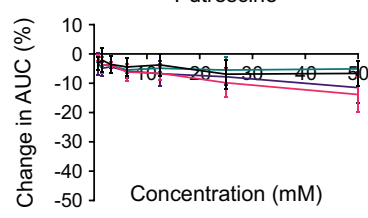

- Standard $=\mathrm{C}$ limitation

C

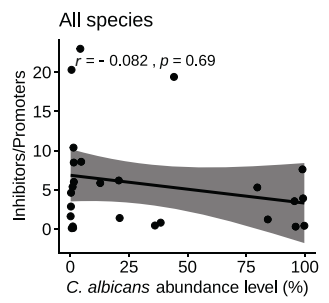

deoxy-Adenosine

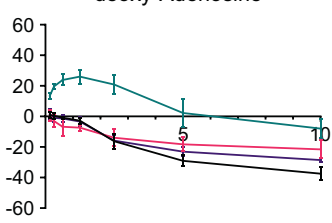

L-Proline

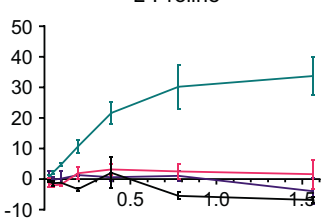

Formate

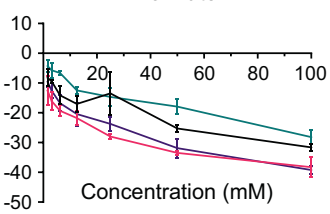

$-\mathrm{N}$ limitation $-\mathrm{C} / \mathrm{N}$ low

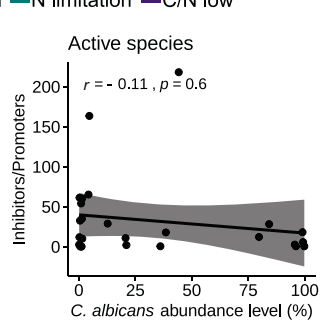

Fig. 3 Experimental and clinical data supporting in silico predictions. A Area under the curve (AUC) measurements for fungal growth in presence of selected metabolites in a series of concentration dilution experiments. AUCs were determined for three replicates. Mean change compared to medium-only controls is shown with standard deviations (SD) as error bars. B Bacterial abundance and growth rates were obtained using MetaPhlAn2 and GRiD 1.2, respectively. Modeled species were arranged according to the Open Tree of Life 10.4 [34]. Annotation rings from inner to outer: Significant correlations between C. albicans abundance and bacterial abundance (magenta stars) or bacterial GRiD score (green stars, Spearman's coefficient, $p<0.05$ ); species with GRiD score greater than 1 in at least one sample (black triangles); in silico interaction coefficients from GSMM analysis (blue

B

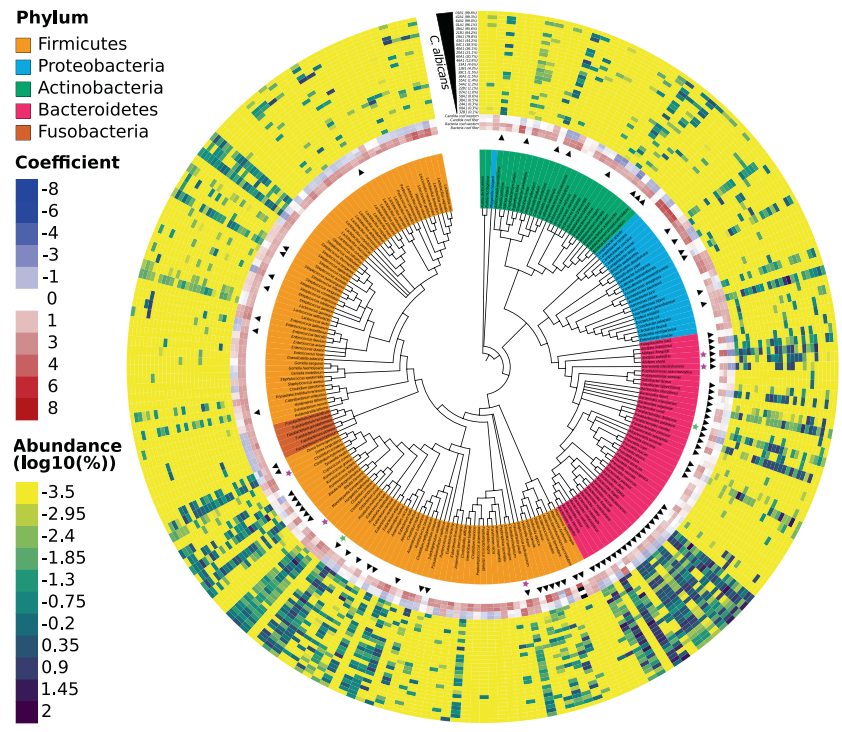

D
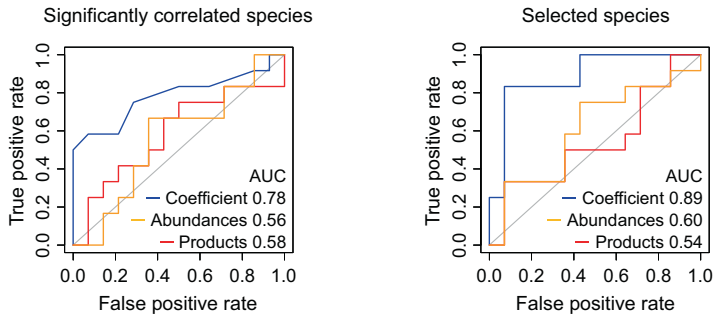

to red); sample bacterial abundance ( $N=26$, yellow to purple) sorted by $C$. albicans abundance (highest abundance is outermost ring). $\mathbf{C}$ Regression performance for all (left panel) or active (GRiD value $>1$ in at least one patient, right panel) species. Each dot is a ratio of inhibitors to promoters for a patient. Values for inhibitors and promoters were calculated by summing products of bacterial abundance $\mathrm{x}$ in silico coefficient for $C$. albicans. D Area under the curve (AUC) of ordinal regression analysis for seven species with significant correlations with $C$. albicans abundance or GRiD values as shown in $\mathbf{B}$ (left panel). AUC of ordinal regression analysis for 29 selected species (see main text). The regression model performance was achieved by using GSMM based metabolic coefficients (Coefficients), bacterial relative abundance (Abundances) or the product of both (Products). 
L-Proline showed concentration-dependent fungal growthpromoting effects specifically under nitrogen limitation. For both metabolites, we predicted a net consumption by $C$. albicans growth inhibiting bacteria. It is noteworthy that, while we did not test for morphological changes, proline and putrescine are known inducers of hyphae formation in C. albicans, which may therefore be influenced by bacterial production and consumption of these metabolites [46-48]. Interestingly, desoxy-adenosine showed a growthpromoting effect at low concentrations, most pronounced under nitrogen limitation, whereas higher metabolite concentrations had negative effects on $C$. albicans growth. In our in silico model we predicted its production by $C$. albicans growth inhibting bacteria. A bacterial production of desoxy-adenosine may therefore lead to sufficiently high concentration levels of this metabolite that might restrict growth of the fungus. In summary, our in silico analysis of metabolite exchanges between fungus and bacteria and the shown experimental data provide a concept for predicting and testing potentially fungal growth modulating metabolites.

Next, we investigated whether metabolic interactions are the main driver of the observed abundance-based associations of gut bacteria and $C$. albicans by analyzing stool samples from a cohort of 24 cancer patients (Supplementary Table S8). We assessed the structure of the gut microbiome in samples via shotgun metagenomic sequencing, generating $118.1 \mathrm{Gbp}$ of sequencing data with an average of $2 \times$ 26.2 million paired-end reads per sample. Taxonomic profiling revealed that Bacteroidetes (54.3\%) and Firmicutes $(36.3 \%)$ were the most abundant phyla, followed by Proteobacteria $(5.83 \%)$, Verrucomicrobia $(1.46 \%)$ and Actinobacteria $(1.34 \%)$. From the 400 bacterial species identified in our samples, we retrieved GSMMs for 247 (Fig. 3B, Supplementary Fig. S2). The mean relative abundance of $C$. albicans within the fungal community as revealed by ITS1 amplicon sequencing of all cancer patient samples was $39.7 \%$ over all patient samples, while individual samples covered a broad relative abundance range from $0.1 \%$ to $99.8 \%$ relative $C$. albicans abundance. Only five bacterial species had a relative abundance with a significant correlation by Spearman's coefficient to C. albicans relative abundance across all patients (Clostridium scindens $\rho=0.45$, Hungatella hathewayi $\rho=0.55$, Flavonifacfractor plautii $\rho=0.46$, Barnesiella intestinihominis $\rho=-0.47$ and Alistipes putredinis $\rho=-0.42$, Fig. 3B). Although the influence of external factors such as gender, age or therapy type cannot be completely ruled out, this suggests that, at least in terms of relative abundance compared to other fungal species, a limited set of gut microbes might influence C. albicans prevalence levels more than the remaining gut microbiome constituting bacteria. Of note, the directionality of the observed significant correlation was in accordance with the predicted paired-growth relationship by the GSMMs for all five bacteria (Supplementary Table S1). We also calculated Growth Rate InDex (GRiD) [49] to estimate in situ growth rates for bacteria species and analyze correlation to $C$. albicans relative abundance. We observed a finite GRiD value for 98 of the species in Fig. 3B (cf. Supplementary Table S9). GRiD values for two bacteria significantly correlated with $C$. albicans abundance (positive for Roseburia inulinivorans and negative for Parabacteroides distasonis, Fig. 3B) in the same direction as predicted by our GSMMs.

We next investigated to what extent fungal growth is influenced by spent media of selected bacterial strains for which we established cultivation protocols in our lab (Methods). Among the species selected for in vitro spent media experiments, A. putredinis had the strongest inhibitory effect in Western-diet conditions in in silico predictions (Supplementary Table S1). This species was also found significantly negatively correlated with $C$. albicans abundance in the human gut (Fig. 3B). Of note, adjusting for the external factors gender, age, ethnicity or immunotherapy application in our metagenomic dataset, affected this significant negative correlation only slightly (Supplementary Table S8). The addition of spent media from A. putredinis to $C$. albicans cultures (cf. Methods for details) resulted in up to 23\% growth inhibition of the fungus (Supplementary Table S2). Interestingly, both butanoic and propanoic acid, two short chain fatty acids (SCFAs) with fungistatic properties produced by the gut microbiome $[50,51]$ were found with slight elevated concentrations in the spent media of $A$. putredinis $(202 \mu \mathrm{M}$ and $116 \mu \mathrm{M}$ compared to $148 \mu \mathrm{M}$ and $88 \mu \mathrm{M}$ in modified GAM for propanoic and butanoic acid, respectively, Supplementary Table S2). A. putredinis is a reported producer of propionate presumably next to succinate [51]. Of note, for pairwise in silico simulations over all possible combinations of $A$. putredinis and any bacteria that are present in our human samples, we predicted that in 63\% of the paired models A. putredinis can secrete propanoic acid. These data indicate that to a certain extend A. putredinis might contribute to global propanoic but also butanoic acid levels, two health promoting SCFAs [52]. In addition, A. putredinis is dominant in the fecal microbiota of healthy humans [53] and important for the maintenance of a healthy intestinal barrier [54]. Generally, the genus Alistipes shows disease protective effects against a number of diseases including fibrosis, colitis, cancer or cardiovascular disease [55]. Our results hint that A. putredinis is also potentially able to prevent elevated $C$. albicans levels, and we suggest more in-depth studies of $A$. putredinis in conjunction with C. albicans. To further examine whether the observed inhibition was a methodological artifact we performed additional experiments with species showing either positive or negative interaction with $C$. albicans. For example, our 
in silico predictions show that Ruminococcus torques contributes positively to $C$. albicans growth, while also a nonsignificant positive correlation between this species and $C$. albicans levels was observed from the metagenomics data (Fig. 3B, Supplementary Table S8). Indeed, in our spent media experiment we also observed a positive effect of Ruminococcus torques on C. albicans growth (15\% for $100 \%$ spent media, $12 \%$ for $75 \%$ spent media, Supplementary Table S2). Other strains showed varied levels of $C$. albicans growth inhibition which is in agreement with our metagenomic and spent media data except for Escherichia coli. For E. coli we observe a disagreement between a predicted positive growth effect on $C$. albicans, a low positive correlation in the metagenomics data, and a negative effect in the spent media experiments. Interestingly, $E$. coli was found to produce a soluble fungicidal factor, which cannot be captured by our metabolic model and might explain the discordance to our spent media results [56].

To further support the idea that metabolic interactions with a few bacterial species might be sufficient to determine C. albicans colonization levels, we investigated, by ordinal regression analysis, if the ratio of abundance $\mathrm{x}$ growth coefficients for all bacterial inhibitors vs. promoters correlated with $C$. albicans levels (Methods, Ordinal regression model). No significant correlation was seen when considering all species (Fig. 3C, left), indicating that the use of all species as predictors does not allow for a good model to predict $C$. albicans levels. Of note, the ratio of inhibitors to promoters calculated using only species that were active in at least one patient sample according to GRiD was not significantly correlated to $C$. albicans abundance levels as well (Fig. 3C, right). The critical role of metabolic interactions between the limited set of 7 significantly correlating bacterial species identified above to $C$. albicans relative abundances was evident in the ordinal regression model we developed using the interaction growth coefficients and relative abundances of the bacteria. We investigated if GSMM computed growth coefficients, relative bacterial abundance, or both were good predictors for C. albicans levels (Method, Ordinal regression models). We obtained the highest performance of 0.78 Area Under the Receiver Operating Characteristics (AUROC) using growth prediction coefficients from our GSMM computation (Fig. 3D, left). Using only relative bacteria abundance resulted in 0.56 AUROC, whereas a model using both interaction coefficients and growth data resulted in 0.58 AUROC. In addition to using bacteria that show significant correlations to $C$. albicans relative abundances as model features, we filtered candidate bacteria by discarding first the bacteria prevalent in at least $10 \%$, but not more than $90 \%$ of the samples. This reduces the number of feature candidates to 121 bacteria. We selected next 10 subgroups of our samples by ignoring $\sim 10 \%$ samples in each subset, such that each sample was once not part of the subset. For each subset we started with one bacterium and assessed model performance consecutively by adding further bacteria until all bacteria were included as features. Bacteria that caused a drop in model accuracy or regression slope were discarded, followed by another iteration of the model performance evaluation. These steps were repeated until no performance-impairing bacteria were left in the feature list. The union of the gained feature candidates across all 10 subsets resulted in a feature list of 57 gut bacteria, which included, among others, A. putridinis (Supplementary Table S10). We evaluated the regression model with these bacteria and obtained 0.89 AUROC using only growth prediction coefficients from our GSMM computation (Fig. 3D, right). Using only relative bacteria abundance or both, interaction coefficients and growth data, resulted again in a performance drop down to 0.60 and 0.54 AUROC, respectively. These results demonstrate that GSMM analysis based interaction coefficients could be used as predictors of ordinally scaled C. albicans levels. Our results indicate that the intrinsic metabolic interactivity between a fungus and bacteria contains valuable information about the performance of classification models. This information should be more extensively applied in future classification studies.

In conclusion we expanded the concept of using in silico metabolic interaction calculations to accurately predict pairwise beneficial or detrimental effects on co-existing organisms [18, 25, 57] to bacterial-fungal interaction predictions based on studies suggesting that key gut species might determine beneficial outcomes in patients with a range of diseases [5, 6, 9]. Selected metabolite experiments and shotgun metagenomics sequencing back our in silico modeling concept based on pairwise metabolic interaction simulations. Further studies of our predictions for major metabolic pathways, for example for carbon compounds and amino acids, may elucidate the specific mechanisms of these influences. Selected metabolite measurements in defined media could be used further for accurate predictions of potential metabolite candidates that are preferentially used by e.g., gut microbes over $C$. albicans and can hint towards bacterial species that specifically secrete $C$. albicans-inhibiting metabolites. Taken together, our findings support that specific gut bacteria influence gut colonization by $C$. albicans. Moreover, our analysis indicates that it may be possible to design synthetic communities with only a few bacterial species that could then influence essential metabolic activities of $C$. albicans and prevent fungal overgrowth. Further refinements of our model including compartmentalization complemented by comprehensive single-knockout studies of bacteria or the fungus may further improve the predictive capacity. Also additional diets and growth media compositions beyond the three used in this study may be tested, since e.g., GAM compositions 
may vary, therefore influence in silico predictions and in general, might not reflect in vivo conditions as well as high fiber, western or other common human diets. Despite pairwise interactions were shown to be key drivers of the dynamics of microbial communities [58], additional in silico simulations of multiple interactions with e.g., the recently published MAMBO algorithm [59] need to be addressed, to potentially extend our understanding of the intricate relationship between $C$. albicans and the gut microbiota and its effect on C. albicans levels. Finally, tools that incorporate spatial information [60, 61] could determine the impact of niche colonization by gut fungal and bacterial species. In the present study we specifically focused on the intricate relationship between (gut) bacteria and $C$. albicans to elucidate their relationship independent of host factors in order to keep free parameters in a feasible range. Host factors are key modulators of fungal-bacterial or fungal-bacterial-host interactions as could be shown in other studies [3, 62-64]. Though adding considerable complexity to the setup, an extension of our conceptual approach to study specifically metabolic modes of fungal-bacterial interaction with host factors might play an important complementary role as long as the predictive capacity can be supported by sufficient data around the triangle host, bacteria and fungus. In summary, our strategy of studying fungal-bacterial relationships in the gut using an in silico, metabolism-driven approach already yielded promising results. Our approach adds another useful layer of in silico predictions that can contribute to stratify identification of potentially clinically relevant gut bacteria in face of the steadily growing amount of high throughput data. Ultimately, including metabolic in silico analysis could promote additional systems-biology and systemsmedicine studies that focus on fungal infections and their often lethal implications to humans.

Acknowledgements GP, MM, SV and PB would like to thank Deutsche Forschungsgemeinschaft (DFG) CRC/Transregio 124 "Pathogenic fungi and their human host: Networks of interaction", subproject B5 and C2 (DFG project number 210879364). RVU, FSdOL acknowledge funding from The Novo Nordisk Foundation under NFF grant number: NNF10CC1016517 and The Novo Nordisk Foundation, Challenge programme, CaMiT under grant agreement: NNF17CO0028232. We also acknowledge funding by the German Ministry for Education and Science in the program Unternehmen Region (BMBF 03Z22JN11). We thank the patients for providing their stool samples and Chris Tachibana for language revision of our manuscript.

\section{Compliance with ethical standards}

Conflict of interest GJW is an employee of SOTIO and former employee of Unum Therapeutics, reports personal fees from MiRanostics Consulting, Paradigm, Genomic Health, Angiex, Imaging Endpoints II, IBEX Medical Analytics, Spring Bank Pharmaceuticals, Pfizer, IDEA Pharma, GLG Council, Guidepoint Global, Ignyta, and
Circulogene; has received travel reimbursement from Cambridge HealthTech Institute, GlaxoSmith Kline, and Tesaro; has ownership interest in MiRanostics Consulting, Exact Sciences, and Circulogene; and has a patent for methods and kits to predict prognostic and therapeutic outcome in small cell lung cancer issued. All other authors declare no competing interests.

Publisher's note Springer Nature remains neutral with regard to jurisdictional claims in published maps and institutional affiliations.

Open Access This article is licensed under a Creative Commons Attribution 4.0 International License, which permits use, sharing, adaptation, distribution and reproduction in any medium or format, as long as you give appropriate credit to the original author(s) and the source, provide a link to the Creative Commons license, and indicate if changes were made. The images or other third party material in this article are included in the article's Creative Commons license, unless indicated otherwise in a credit line to the material. If material is not included in the article's Creative Commons license and your intended use is not permitted by statutory regulation or exceeds the permitted use, you will need to obtain permission directly from the copyright holder. To view a copy of this license, visit http://creativecommons. org/licenses/by/4.0/.

\section{References}

1. Kullberg BJ, Arendrup MC. Invasive Candidiasis. N Engl J Med. 2015;373:1445-56.

2. Perlroth J, Choi B, Spellberg B. Nosocomial fungal infections: epidemiology, diagnosis, and treatment. Med Mycol. 2007;45: 321-46.

3. Fan D, Coughlin LA, Neubauer MM, Kim J, Kim MS, Zhan X, et al. Activation of HIF- $1 \alpha$ and LL-37 by commensal bacteria inhibits Candida albicans colonization. Nat Med. 2015;21:808-14.

4. Brown GD, Denning DW, Gow NAR, Levitz SM, Netea MG, White TC. Hidden killers: human fungal infections. Sci Transl Med. 2012;4:165rv13.

5. Förster TM, Mogavero S, Dräger A, Graf K, Polke M, Jacobsen ID, et al. Enemies and brothers in arms: Candida albicans and gram-positive bacteria. Cell Microbiol. 2016;18:1709-15.

6. da Silva Dantas A, Lee KK, Raziunaite I, Schaefer K, Wagener J, Yadav B, et al. Cell biology of Candida albicans-host interactions. Curr Opin Microbiol. 2016;34:111-8.

7. Zelante T, Iannitti RG, Cunha C, De Luca A, Giovannini G, Pieraccini G, et al. Tryptophan catabolites from microbiota engage aryl hydrocarbon receptor and balance mucosal reactivity via interleukin-22. Immunity. 2013;39:372-85.

8. Kaewsrichan J, Peeyananjarassri K, Kongprasertkit J. Selection and identification of anaerobic lactobacilli producing inhibitory compounds against vaginal pathogens. FEMS Immunol Med Microbiol. 2006;48:75-83.

9. Kumar S, Bansal A, Chakrabarti A, Singhi S. Evaluation of efficacy of probiotics in prevention of candida colonization in a PICU-a randomized controlled trial. Crit Care Med. 2013;41:565-72.

10. Feist AM, Henry CS, Reed JL, Krummenacker M, Joyce AR, Karp PD, et al. A genome-scale metabolic reconstruction for Escherichia coli K-12 MG1655 that accounts for 1260 ORFs and thermodynamic information. Mol Syst Biol. 2007;3:121.

11. Feist AM, Palsson B $\emptyset$. The growing scope of applications of genome-scale metabolic reconstructions using Escherichia coli. Nat Biotechnol. 2008;26:659-67.

12. Trinh CT, Srienc F. Metabolic engineering of Escherichia coli for efficient conversion of glycerol to ethanol. Appl Environ Microbiol. 2009;75:6696-705. 
13. Gomes de Oliveira Dal'Molin C, Quek L-E, Saa PA, Nielsen LK. A multi-tissue genome-scale metabolic modeling framework for the analysis of whole plant systems. Front Plant Sci. 2015;6:4.

14. van Hoek MJA, Merks RMH. Redox balance is key to explaining full vs. partial switching to low-yield metabolism. BMC Syst Biol. 2012;6:22.

15. Pfeiffer T, Morley A. An evolutionary perspective on the Crabtree effect. Front Mol Biosci. 2014;1:17.

16. Nilsson A, Nielsen J. Metabolic trade-offs in yeast are caused by F1F0-ATP synthase. Sci Rep. 2016;6:22264.

17. Shlomi T, Benyamini T, Gottlieb E, Sharan R, Ruppin E. Genome-scale metabolic modeling elucidates the role of proliferative adaptation in causing the Warburg effect. PLoS Comput Biol. 2011;7:e1002018.

18. Magnúsdóttir S, Heinken A, Kutt L, Ravcheev DA, Bauer E, Noronha A, et al. Generation of genome-scale metabolic reconstructions for 773 members of the human gut microbiota. Nat Biotechnol. 2016;35:81-9.

19. Machado D, Andrejev S, Tramontano M, Patil KR. Fast automated reconstruction of genome-scale metabolic models for microbial species and communities. Nucleic Acids Res. 2018;46:7542-53.

20. Castillo S, Barth D, Arvas M, Pakula TM, Pitkänen E, Blomberg P, et al. Whole-genome metabolic model of Trichoderma reesei built by comparative reconstruction. Biotechnol Biofuels. 2016; 9:252.

21. Pitkänen E, Jouhten P, Hou J, Syed MF, Blomberg P, Kludas J, et al. Comparative genome-scale reconstruction of gapless metabolic networks for present and ancestral species. PLoS Comput Biol. 2014;10:e1003465.

22. Ene IV, Lohse MB, Vladu AV, Morschhäuser J, Johnson AD, Bennett RJ. Phenotypic profiling reveals that Candida albicans opaque cells represent a metabolically specialized cell state compared to default white cells. mBio. 2016;7:e01269-16.

23. Fritzemeier CJ, Hartleb D, Szappanos B, Papp B, Lercher MJ. Erroneous energy-generating cycles in published genome scale metabolic networks: Identification and removal. PLOS Comput Biol. 2017;13:e1005494.

24. Mahadevan R, Schilling $\mathrm{CH}$. The effects of alternate optimal solutions in constraint-based genome-scale metabolic models. Metab Eng. 2003;5:264-76.

25. Heinken A, Thiele I. Systems biology of host-microbe metabolomics. Wiley Interdiscip Rev. 2015;7:195-219.

26. Vaas LAI, Sikorski J, Hofner B, Fiebig A, Buddruhs N, Klenk HP, et al. Opm: an R package for analysing OmniLog ${ }^{\circledR}$ phenotype microarray data. Bioinformatics. 2013;29:1823-4.

27. Qin J, Li Y, Cai Z, Li S, Zhu J, Zhang F, et al. A metagenomewide association study of gut microbiota in type 2 diabetes. Nature. 2012;490:55-60.

28. Truong DT, Franzosa EA, Tickle TL, Scholz M, Weingart G, Pasolli E, et al. MetaPhlAn2 for enhanced metagenomic taxonomic profiling. Nat Methods. 2015;12:902-3.

29. Li J, Sung CYJ, Lee N, Ni Y, Pihlajamäki J, Panagiotou G, et al. Probiotics modulated gut microbiota suppresses hepatocellular carcinoma growth in mice. Proc Natl Acad Sci USA. 2016;113: E1306-15.

30. Callahan BJ, McMurdie PJ, Rosen MJ, Han AW, Johnson AJA, Holmes SP. DADA2: High-resolution sample inference from Illumina amplicon data. Nat Methods. 2016;13:581-3.

31. Schloss PD, Westcott SL, Ryabin T, Hall JR, Hartmann M, Hollister EB, et al. Introducing mothur: open-source, platformindependent, community-supported software for describing and comparing microbial communities. Appl Environ Microbiol. 2009;75:7537-41.

32. Caporaso JG, Kuczynski J, Stombaugh J, Bittinger K, Bushman FD, Costello EK, et al. QIIME allows analysis of high-throughput community sequencing data. Nat Methods. 2010;7:335-6.
33. Kõljalg U, Nilsson RH, Abarenkov K, Tedersoo L, Taylor AFS, Bahram M, et al. Towards a unified paradigm for sequence-based identification of fungi. Mol Ecol. 2013;22:5271-7.

34. Hinchliff CE, Smith SA, Allman JF, Burleigh JG, Chaudhary R, Coghill LM, et al. Synthesis of phylogeny and taxonomy into a comprehensive tree of life. Proc Natl Acad Sci USA. 2015;112: 12764-9.

35. Herrgård MJ, Swainston N, Dobson P, Dunn WB, Arga KY, Arvas $\mathrm{M}$, et al. A consensus yeast metabolic network reconstruction obtained from a community approach to systems biology. Nat Biotechnol. 2008;26:1155-60.

36. Aung HW, Henry SA, Walker LP. Revising the representation of fatty acid, glycerolipid, and glycerophospholipid metabolism in the consensus model of yeast metabolism. Ind Biotechnol. 2013; 9:215-28.

37. Tramontano M, Andrejev S, Pruteanu M, Klünemann M, Kuhn M, Galardini $\mathrm{M}$, et al. Nutritional preferences of human gut bacteria reveal their metabolic idiosyncrasies. Nat Microbiol. 2018;3:514-22.

38. Orth JD, Thiele I, Palsson BØ. What is flux balance analysis? Nat Biotechnol. 2010;28:245-8.

39. Lewis NE, Hixson KK, Conrad TM, Lerman JA, Charusanti P, Polpitiya AD, et al. Omic data from evolved E. coli are consistent with computed optimal growth from genome-scale models. Mol Syst Biol. 2010;6:390.

40. Askew C, Sellam A, Epp E, Hogues H, Mullick A, Nantel A, et al. Transcriptional Regulation of Carbohydrate Metabolism in the Human Pathogen Candida albicans. PLoS Pathog. 2009;5: e1000612.

41. Kraidlova L, Schrevens S, Tournu H, Van Zeebroeck G, Sychrova $\mathrm{H}$, Van Dijck P, et al. Characterization of the Candida albicans amino acid permease family: Gap2 is the only general amino acid permease and Gap4 Is an S-Adenosylmethionine (SAM) transporter required for SAM-induced morphogenesis. mSphere. 2016;1:e00284-16.

42. Brunke S, Seider K, Richter ME, Bremer-Streck S, Ramachandra $\mathrm{S}$, Kiehntopf $\mathrm{M}$, et al. Histidine degradation via an aminotransferase increases the nutritional flexibility of Candida glabrata. Eukaryot Cell. 2014;13:758-65.

43. Rząd K, Milewski S, Gabriel I. Versatility of putative aromatic aminotransferases from Candida albicans. Fungal Genet Biol. 2018;110:26-37.

44. Mardinoglu A, Shoaie S, Bergentall M, Ghaffari P, Zhang C, Larsson E, et al. The gut microbiota modulates host amino acid and glutathione metabolism in mice. Mol Syst Biol. 2015;11:834.

45. Jastrzębowska K, Gabriel I. Inhibitors of amino acids biosynthesis as antifungal agents. Amino Acids. 2015;47:227-49.

46. Dabrowa N, Taxer SSS, Howard DH. Germination of Candida albicans induced by proline. Infect Immun. 1976;13:830-5.

47. Silao FGS, Ward M, Ryman K, Wallström A, Brindefalk B, Udekwu K, et al. Mitochondrial proline catabolism activates Ras1/ cAMP/PKA-induced filamentation in Candida albicans. PLoS Genet. 2019;15:e1007976.

48. Herrero AB, López MC, García S, Schmidt A, Spaltmann F, Ruiz-Herrera $J$, et al. Control of filament formation in Candida albicans by polyamine levels. Infect Immun. 1999;67:4870-8.

49. Emiola A, Oh J. High throughput in situ metagenomic measurement of bacterial replication at ultra-low sequencing coverage. Nat Commun. 2018;9:4956.

50. Cottier F, Tan ASM, Xu X, Wang Y, Pavelka N. MIG1 Regulates Resistance of Candida albicans against the Fungistatic Effect of Weak Organic Acids. Eukaryot Cell. 2015;14:1054-61.

51. Louis P, Flint HJ. Formation of propionate and butyrate by the human colonic microbiota. Environ Microbiol. 2017;19:29-41.

52. Morrison DJ, Preston T. Formation of short chain fatty acids by the gut microbiota and their impact on human metabolism. Gut Microbes. 2016;7:189-200. 
53. Tap J, Mondot S, Levenez F, Pelletier E, Caron C, Furet J-P, et al. Towards the human intestinal microbiota phylogenetic core. Environ Microbiol. 2009;11:2574-84.

54. Kuhn KA, Schulz HM, Regner EH, Severs EL, Hendrickson JD, Mehta G, et al. Bacteroidales recruit IL-6-producing intraepithelial lymphocytes in the colon to promote barrier integrity. Mucosal Immunol. 2018;11:357-68.

55. Parker BJ, Wearsch PA, Veloo ACM, Rodriguez-Palacios A. The genus alistipes: gut bacteria with emerging implications to inflammation, cancer, and mental health. Front Immunol 2020;11:906.

56. Cabral DJ, Penumutchu S, Norris C, Morones-Ramirez JR, Belenky P. Microbial competition between escherichia coli and candida albicans reveals a soluble fungicidal factor. Microb Cell. 2018;5:249-55.

57. Heinken A, Sahoo S, Fleming RMT, Thiele I. Systems-level characterization of a host-microbe metabolic symbiosis in the mammalian gut. Gut Microbes. 2013;4:28-40.

58. Venturelli OS, Carr AV, Fisher G, Hsu RH, Lau R, Bowen BP, et al. Deciphering microbial interactions in synthetic human gut microbiome communities. Mol Syst Biol. 2018;14.

59. Garza DR, Verk MC, Huynen MA, Dutilh BE. Towards predicting the environmental metabolome from metagenomics with a mechanistic model. Nat Microbiol. 2018;3:456-60.

60. Biggs MB, Papin JA. Novel multiscale modeling tool applied to pseudomonas aeruginosa biofilm formation. PLoS One. 2013;8: e78011.

61. Bauer E, Zimmermann J, Baldini F, Thiele I, Kaleta C. BacArena: Individual-based metabolic modeling of heterogeneous microbes in complex communities. PLOS Comput Biol. 2017;13:e1005544.
62. Liang SH, Anderson MZ, Hirakawa MP, Wang JM, Frazer C, Alaalm LM, et al. Hemizygosity enables a mutational transition governing fungal virulence and commensalism. Cell Host Microbe. 2019;25:418-31.e6.

63. Allert S, Förster TM, Svensson CM, Richardson JP, Pawlik T, Hebecker B, et al. Candida albicans-induced epithelial damage mediates translocation through intestinal barriers. mBio. 2018;9: e00915-18.

64. Leonardi I, Li X, Semon A, Li D, Doron I, Putzel G, et al. CX3CR1+, mononuclear phagocytes control immunity to intestinal fungi. Science. 2018;359:232-6.

65. Gillum AM, Tsay EY, Kirsch DR. Isolation of the Candida albicans gene for orotidine-5'-phosphate decarboxylase by complementation of S. cerevisiae ura3 and E. coli pyrF mutations. Mol Gen Genet. 1984;198:179-82.

66. Chauvel M, Nesseir A, Cabral V, Znaidi S, Goyard S, BachellierBassi S, et al. A Versatile overexpression strategy in the pathogenic Yeast Candida albicans: Identification of regulators of morphogenesis and fitness. PLoS ONE 2012;7:e45912.

67. Noble SM, Johnson AD. Strains and strategies for large-scale gene deletion studies of the diploid human fungal pathogen candida albicans. Eukaryotic Cell 2005;4:298-309.

68. Homann OR, Dea J, Noble SM, Johnson AD. A Phenotypic profile of the candida albicans regulatory network. PLoS Genet 2009;5:e1000783.

69. Jiménez-López C, Collette JR, Brothers KM, Shepardson KM, Cramer RA, Wheeler RT, et al. Candida albicans induces arginine biosynthetic genes in response to host-derived reactive oxygen species. Eukaryotic Cell 2012;12:91-100. 